\title{
DERIVED SCHWARZ MAP OF THE HYPERGEOMETRIC DIFFERENTIAL EQUATION AND A PARALLEL FAMILY OF FLAT FRONTS
}

\author{
TAKESHI SASAKI, KOTARO YAMADA, AND MASAAKI YOSHIDA
}

\begin{abstract}
In the paper 7 we defined a map, called the hyperbolic Schwarz map, from the one-dimensional projective space to the three-dimensional hyperbolic space by use of solutions of the hypergeometric differential equation, and thus obtained closed flat surfaces belonging to the class of flat fronts. We continue the study of such flat fronts in this paper. First, we introduce the notion of derived Schwarz maps of the hypergeometric differential equation and, second, we construct a parallel family of flat fronts connecting the classical Schwarz map and the derived Schwarz map.
\end{abstract}

\section{INTRODUCTION}

Consider the hypergeometric differential equation

$E(a, b, c) \quad x(1-x) u^{\prime \prime}+\{c-(a+b+1) x\} u^{\prime}-a b u=0$, and define its Schwarz map as a multi-valued map on $X=C-\{0,1\}$ by

$$
S: X \ni x \longmapsto u_{0}(x): u_{1}(x) \in \boldsymbol{P}^{1},
$$

where $u_{0}$ and $u_{1}$ are linearly independent solutions of $E(a, b, c)$ and $\boldsymbol{P}^{1}$ is the complex projective line. A change of the unknown $u$ by multiplying a non-zero function, takes the equation into the SL-form:

$$
u^{\prime \prime}-q(x) u=0
$$

The coefficient $q$ is expressed as

$$
q=-\{S ; x\}
$$

where $\{S ; x\}$ is the Schwarzian derivative

$$
\{S ; x\}:=\frac{1}{2}\left(\frac{S^{\prime \prime}}{S^{\prime}}\right)^{\prime}-\frac{1}{4}\left(\frac{S^{\prime \prime}}{S^{\prime}}\right)^{2} .
$$

For two linearly independent solutions $u_{0}$ and $u_{1}$ to this equation, we define the derived Schwarz map

$$
D S: X \ni x \longmapsto u_{0}^{\prime}(x): u_{1}^{\prime}(x) \in \boldsymbol{P}^{1},
$$

and the hyperbolic Schwarz map

$$
H S: X \ni x \longmapsto H(x)=U(x){ }^{t} \bar{U}(x) \in \boldsymbol{H}^{3},
$$

where

$$
U=\left(\begin{array}{ll}
u_{0} & u_{0}^{\prime} \\
u_{1} & u_{1}^{\prime}
\end{array}\right)
$$

Date: February 28, 2007.

2000 Mathematics Subject Classification. 33C05, 53C42.

Key words and phrases. hypergeometric differential equation, Schwarz map, hyperbolic Schwarz map, derived Schwarz map, flat front. 
and $\boldsymbol{H}^{3}$ is the hyperbolic 3 -space identified with the space of positive $2 \times 2$-hermitian matrices modulo diagonal ones. The hyperbolic Schwarz map is considered as a flat front in $\boldsymbol{H}^{3}$ in the sense of [5, that is, a flat surface of certain kind of singularities. See Section 2.2 and Section 3.2 for details. We regard $\boldsymbol{P}^{1}$ as the ideal boundary $\partial \boldsymbol{H}^{3}$ of $\boldsymbol{H}^{3}$. Then $S$ and $D S: X \rightarrow \boldsymbol{P}^{1}=\partial \boldsymbol{H}^{3}$ are considered as the two hyperbolic Gauss maps of the flat front $H S$.

We assume the parameters $a, b$ and $c$ are real and satisfy the condition

$$
\left|\mu_{0}\right|, \quad\left|\mu_{1}\right|, \quad\left|\mu_{\infty}\right|<1,
$$

where

$$
\mu_{0}=1-c, \quad \mu_{1}=c-a-b, \quad \mu_{\infty}=b-a
$$

are exponent-differences at 0,1 and $\infty$, respectively. The Schwarz map gives a conformal equivalence between the upper half part

$$
X_{+}:=\{x \in X=C \backslash\{0,1\} \mid \Im(x) \geq 0\}
$$

of $X$ and the image $T:=S\left(X_{+}\right)$, which is bounded by three arcs, a Schwarz triangle. Though the image $D S\left(X_{+}\right)$is bounded by the three circles generated by the three arcs bounding the Schwarz triangle, the situation depends on the parameters $(a, b, c)$. The image surface $H S\left(X_{+}\right)$has, in general, singularities; the situation also depends on the parameters $(a, b, c)$. We study such dependence.

On the other hand, there is a 1-parameter (parallel) family of surfaces (maps from $X$ to $\boldsymbol{H}^{3}$ ) in the hyperbolic 3-space, such that the Schwarz and the derived Schwarz maps are the two extremes (which have the images in $\boldsymbol{P}^{1}$ ), and the hyperbolic Schwarz map is a generic member. For a typical set of parameters, we visualize the 1-parameter family.

\section{DERIVED SCHWARZ MAP}

2.1. Definition of the derived Schwarz map. The equation $E(a, b, c)$ transforms into the SL-form (SL) by the projective change of the unknown

$$
u \longrightarrow \underline{u}:=N \cdot u, \quad \text { where } \quad N:=\sqrt{x^{c}(1-x)^{a+b+1-c}} .
$$

The coefficient $q$ is

$$
\begin{aligned}
q & =-\frac{1}{4}\left\{\frac{1-\mu_{0}^{2}}{x^{2}}+\frac{1-\mu_{1}^{2}}{(1-x)^{2}}+\frac{1+\mu_{\infty}^{2}-\mu_{0}^{2}-\mu_{1}^{2}}{x(1-x)}\right\} \\
& =-\frac{1}{4} \frac{\left(1-\mu_{\infty}^{2}\right) x^{2}+\left(\mu_{\infty}^{2}+\mu_{0}^{2}-\mu_{1}^{2}-1\right) x+1-\mu_{0}^{2}}{x^{2}(1-x)^{2}}
\end{aligned}
$$

Set $v=\underline{u}^{\prime}$; it satisfies the equation

$$
d E(a, b, c) \quad v^{\prime \prime}-\frac{q^{\prime}}{q} v^{\prime}-q v=0 .
$$

The Schwarz map of this equation is called the derived Schwarz map DS of the equation $E(a, b, c)$

2.2. Flat fronts and hyperbolic Gauss maps. Geometrically, the hyperbolic Schwarz map $H S: X_{+} \rightarrow \boldsymbol{H}^{3}$ is a flat front in the sense of [5], and the map $U$ in (1.1) is the holomorphic lift of $H S$, which satisfies the differential equation

$$
U^{-1} U^{\prime}=\left(\begin{array}{ll}
0 & q \\
1 & 0
\end{array}\right) .
$$

That is, under the notations in [5, the canonical forms of the flat front $H S$ are $\omega=d x$, and $\theta=q d x$. From now on, we normalize $U$ as $\operatorname{det} U=1$. 
Though HS may have singularities, the unit normal vector field $\nu$ is well-defined as

$$
\nu=U\left(\begin{array}{rr}
1 & 0 \\
0 & -1
\end{array}\right)^{t} \bar{U}
$$

The hyperbolic Gauss maps $G$ and $G_{*}$ are maps defined as

$$
\begin{aligned}
G(x) & =\left(\begin{array}{l}
\text { the asymptotic class of the geodesic in } \boldsymbol{H}^{3} \\
\text { starting at } H S(x) \text { with initial velocity } \nu(x)
\end{array}\right) \in \partial H^{3}=\boldsymbol{P}^{1} \\
G_{*}(x) & =\left(\begin{array}{l}
\text { the asymptotic class of the geodesic in } \boldsymbol{H}^{3} \\
\text { starting at } H S(x) \text { with initial velocity }-\nu(x)
\end{array}\right) \in \partial H^{3}=\boldsymbol{P}^{1} .
\end{aligned}
$$

The Schwarz map $S$ and the derived Schwarz map $D S$ are nothing but the hyperbolic Gauss maps $G$ and $G_{*}$ respectively. The ramification points of $D S$ are the zeros of $q$, which are the umbilic points of the flat front $H S$.

The isometric action of $\operatorname{PSL}(2, \boldsymbol{C})$ to $\boldsymbol{H}^{3}$ as

$$
\boldsymbol{H}^{3} \ni p \longmapsto a p^{t} \bar{a} \quad( \pm a \in P S L(2, \boldsymbol{C}))
$$

induces the conformal action on $\partial \boldsymbol{H}^{3}=\boldsymbol{P}^{1}$, which coincides with the $\operatorname{PSL}(2, \boldsymbol{C})$ action as the Möbius transformations. Thus, the monodromy representations with respect to $G=S$ and $G_{*}=H S$ coincide.

2.3. Description of the image. Though the maps $S$ and $D S$ are determined by the equation only up to linear fractional transformations, in this section, we always assume that 'for any choice of $S, D S$ is so chosen that if $S=\underline{u}_{1} / \underline{u}_{0}$ then $D S=\underline{u}_{1}^{\prime} / \underline{u}_{0}^{\prime}$.

Lemma 2.1. Under the convention above

$$
S(0)=D S(0), \quad S(1)=D S(1), \quad \text { and } \quad S(\infty)=D S(\infty) .
$$

Proof. Solutions of the SL-form are singular at $x=0,1, \infty$, so de l'Hopital theorem can be applied.

Lemma 2.2. The equation $d E(a, b, c)$ has the same local behavior with $E(a, b, c)$ at the three singular points. In addition, it has apparent singularities at the zeros of $q$. Monodromy behaviors of both equations agree. If the zeros are simple, DS ramifies at these points with index 2 ; if double, with index 3.

Proof. In general, the Schwarzian derivative of any Schwarz map $S$ of an equation $w^{\prime \prime}-Q w=0$ equals $-Q$. From the definition of the Schwarzian derivative, it is straightforward to see that $S$ has the local expression

$$
\left.S=(x-\xi)^{\gamma} \text { (a non-vanishing holomorphic function at } \xi\right)
$$

if and only if $Q$ has the local expression

$$
Q=-\frac{1-\gamma^{2}}{(x-\xi)^{2}}+\frac{\text { a holomorphic function at } \xi}{x-\xi} .
$$

The SL-form of the equation $d E(a, b, c)$ is given by $\underline{v}^{\prime \prime}-\underline{q} \underline{v}=0$, where

$$
\underline{q}=q+\frac{3}{4}\left(\frac{q^{\prime}}{q}\right)^{2}-\frac{1}{2}\left(\frac{q^{\prime \prime}}{q}\right)=q+\frac{1}{4}\left(\frac{q^{\prime}}{q}\right)^{2}-\frac{1}{2}\left(\frac{q^{\prime}}{q}\right)^{\prime} .
$$

If $\alpha$ and $\beta$ denote the zeros of $q$, we have

$$
\frac{q^{\prime}}{q}=-\frac{2}{x}+\frac{2}{1-x}+\frac{1}{x-\alpha}+\frac{1}{x-\beta} .
$$




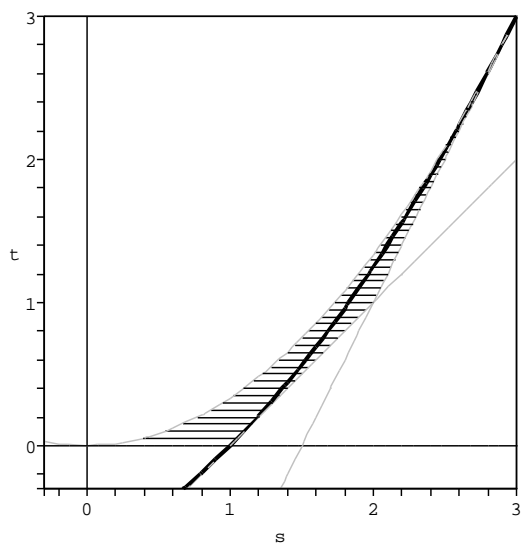

FiguRE 1. Left: Domain of the points $(s, t)$ of the points $(x, y)$ for $s=5 / 3$

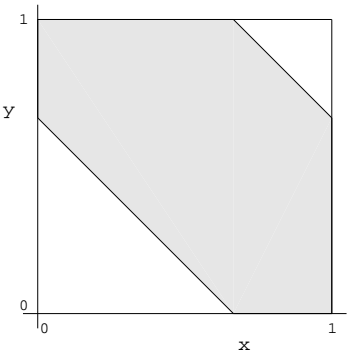

Right: Domain

Since we have

$$
\left(\frac{q^{\prime}}{q}\right)^{2}=\frac{4}{x^{2}}+\frac{O(1)}{x}, \quad\left(\frac{q^{\prime}}{q}\right)^{\prime}=\frac{2}{x^{2}}+\frac{O(1)}{x},
$$

the expression of $\underline{q}$ above leads to

$$
\lim _{x \rightarrow 0} x^{2} q(x)=\lim _{x \rightarrow 0} x^{2} \underline{q}(x) \quad\left(=-\frac{1-\mu_{0}^{2}}{4}\right) ;
$$

this implies that $S$ and $D S$ have the same local behavior at 0 . It can be similarly seen that they have the same local behavior also at 1 and $\infty$. When $\alpha \neq \beta$, by a similar computation, we have

$$
\lim _{x \rightarrow \alpha}(x-\alpha)^{2} \underline{q}(x)=\lim _{x \rightarrow \beta}(x-\beta)^{2} \underline{q}(x)=\frac{1}{4}+\frac{1}{2}=-\frac{1-2^{2}}{4},
$$

and when $\alpha=\beta$,

$$
\lim _{x \rightarrow \alpha}(x-\alpha)^{2} \underline{q}(x)=-\frac{1-3^{2}}{4} .
$$

Remark 2.3. We refer to 8 for a general treatment of differential eqautions that admit apparent singularities in addition to three regular singular points and that the monodoromy groups are triangle groups.

The discriminant of the numerator of $q$ is given as

$$
D:=\left(\mu_{\infty}^{2}+\mu_{0}^{2}-\mu_{1}^{2}-1\right)^{2}-4\left(1-\mu_{0}^{2}\right)\left(1-\mu_{\infty}^{2}\right) .
$$

This quantity turns out to be symmetric with respect to $\left\{\mu_{0}^{2}, \mu_{1}^{2}, \mu_{\infty}^{2}\right\}$, and can be expressed as

$$
\begin{aligned}
& D=(s+1)^{2}-4(t+1), \\
& \text { where } \quad s=\mu_{0}^{2}+\mu_{1}^{2}+\mu_{\infty}^{2}, \quad \text { and } t=\mu_{0}^{2} \mu_{1}^{2}+\mu_{1}^{2} \mu_{\infty}^{2}+\mu_{\infty}^{2} \mu_{0}^{2} .
\end{aligned}
$$

Since we assumed $0 \leq \mu_{0}^{2}, \mu_{1}^{2}, \mu_{\infty}^{2}<1$,

Lemma 2.4. The domain of existence of the point $(s, t)$ is bounded by

$$
t=\frac{s^{2}}{3}, \quad t=0, \quad t=s-1, \quad \text { and } \quad t=2 s-3,
$$

see Figure 1, and is divided into two parts by the curve $D=0$. 
Proof. For notational simplicity, set $s=x+y+z, t=x y+y z+z x$. Substituting $z=s-(x+y)$ into the second expression, we define the function

$$
t_{s}(x, y)=x y+(x+y)\{s-(x+y)\},
$$

where $(x, y)$ is in the region:

$$
0 \leq x, y<1, \quad 0 \leq s-(x+y)<1 .
$$

This region changes its shape at $s=1$ and 2 ; so we consider three cases (1) $0 \leq s \leq 1$, (2) $1 \leq s \leq 2$ (refer to the right picture of Figure 1), (3) $2 \leq s<3$. In any case, the function $t_{s}$ attains its maximum $s^{2} / 3$ at $(s / 3, s / 3)$, while it attains its minima $0, s-1,2 s-3$ at different boundary points of the regions for cases (1), $(2)$, and (3), respectively.

We collect properties of the Schwarz and the derived Schwarz maps, especially their images.

Proposition 2.5. For the real parameters $(a, b, c)$ satisfying the condition (1.2),

(1) $S$ gives a conformal isomorphism from $X_{+}$onto the triangle $T=S\left(X_{+}\right)$,

(2) $D S(x)=S(x)$ for $x=0,1, \infty$,

(3) DS, restricted to the interval $(-\infty, 0)$ gives a diffeomorphsim onto the side $S((-\infty, 0))$ of $T$,

(4) $D S$, restricted to the interval $(1,+\infty)$ gives a diffeomorphsim onto the side $S((1,+\infty))$ of $T$

(5) the image $D S((0,1))$ is, as a set, the whole circle $C$ extending the arc side $S((0,1))$,

(6) the image $D S\left(X_{+}\right)$is, counting multiplicity, the union of $T$ and the disc (left side of $S((0,1))$ ) bounded by $C$,

(7) if the set of parameters is in the domain $D<0$, then the derived Schwarz map $D S$ has a unique ramification point (of order 2) in the interior of $X_{+}$, and as $x$ moves from 0 to $1, D S(x)$ moves from $S(0)$ along the circle $C$, in the same sense as $S(x)$ moves from $S(0)$, turns around once the circle and reach at $S(1)$.

(8) if the set of parameters is in the domain $D>0$, then $D S$ has two ramification points (of order 2 ) on the interval $(0,1)$, say $r_{1}<r_{2}$, and as $x$ moves from 0 to $1, D S(x)$ moves from $S(0)$ along the circle $C$, in the same sense as $S(x)$ moves from $S(0)$, passes through $S(1)$ and $D S\left(r_{2}\right)$, turns back at $D S\left(r_{1}\right)$ to $D S\left(r_{2}\right)$, turns back at $D S\left(r_{2}\right)$ to $S(0)$ and eventually ends the journey at $S(1)$,

(9) if the set of parameters satisfy $D=0$, then $r_{1}=r_{2}$ is the unique ramification point (of order 3 ) in $X_{+}$; this is the limit case of $[(7)$ and $(8)$.

Proof. The assertion (1) is well-known and (2) is Lemma 2.1. We prove (3)-(9). We first assume $D<0$, and study the images of one of the three intervals, say $(0,1)$ under $S$ and $D S$. Since the local behavior (and the values) of $S$ and $D S$ coincide at each $x=0,1, \infty$, and since holomorphic maps preserves orientation, as $x$ moves from 0 to $1, D S(x)$ moves from $S(0)$ to $S(1)$ along the circle $C$ in the same sense as $S(x)$ moves from $S(0)$ to $S(1)$. The point $D S(x)$, after leaving $S(0)$ either stops at $S(1)$, or passes through $S(1)$ and turns around the whole circle, passes through $S(0)$ and then stops at $S(1)$, or turns once more, ... The same assertion holds for the other intervals $(-\infty, 0)$ and $(1,+\infty)$.

On the other hand, since there is only one ramification point of degree 2 in $X_{+}$, the rotation number is 2 . Thus two out of the three intervals are mapped by $D S$ to the arc, the image under $S$, and only one interval is mapped to the arc plus the whole circle. In the special dihedral $(n=3)$ case where $\left(\mu_{0}, \mu_{1}, \mu_{\infty}\right)=(1 / 2,1 / 2,1 / 3)$, 

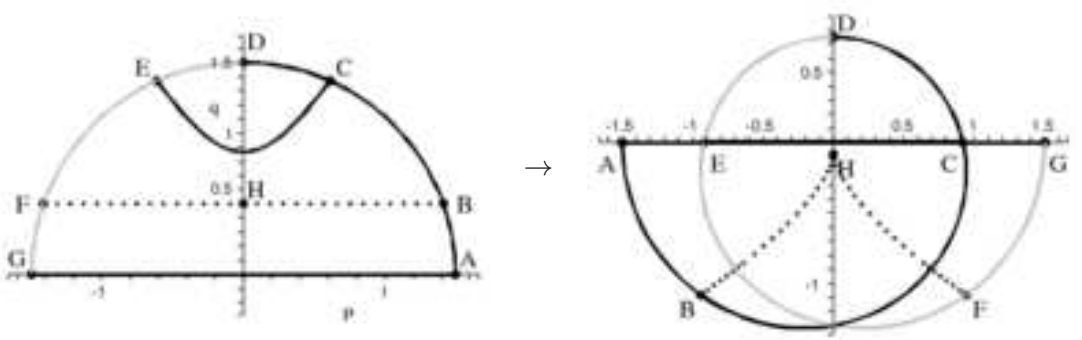

Figure 2. The image of hemi-disc by $x \mapsto z=-x^{3} / 3-x / 4$

it is known that the interval $(0,1)$ plays this part; refer to [7. Since the domain $D<0$ is connected, we conclude that it is always the case.

We next consider what happens when the zeros $\alpha \in \boldsymbol{C}-\boldsymbol{R}$ and $\beta=\bar{\alpha}$ come together $(D=0)$, and then turn to two real roots $(D<0)$. During this process, global behavior of $D S$ does not change. Local behavior around the zeros can be best understood via the following model, by which the proposition is readily proved.

2.4. A model of confluence of the two ramification points of the derived Schwarz map. We see what will happen for the derived Schwarz map when the two complex conjugate ramification points come together and separate into two real points. Such a map (a family of maps) can be locally expressed by

$$
\phi_{t}: C \ni x \mapsto z=-\left(\frac{x^{3}}{3}+t x\right) \in \boldsymbol{C},
$$

where the real parameter $t$ varies from positive to negative. In fact since $z^{\prime}=$ $-\left(x^{2}+t\right)$, the ramification points $\pm i \sqrt{t}$ come together and then separate to $\pm \sqrt{-t}$. Figures 2 and 3 show the images of the upper unit hemi-disc when $t=1 / 4$ and $t=-1 / 4$. Points marked alphabets are mapped to the points with the same marks.

In Figure 2, the curve $\overparen{E C}$ in the left figure is the preimage of the segment $\overline{E C}$ on the real axis in the right figure. It divides the hemi-disc into two regions; the upper one covers once the upper part of the $z$-plane, while the lower one covers twice the lower part. $H$ denotes the ramification point.

In Figure 3. the curves $\overparen{E H}$ and $\overparen{C K}$ in the left figure are the preimages of the line segments $\overline{E H}$ and $\overline{C K}$ on the real axis, respectively. They divide the hemi-disc into three regions; the middle one covers the upper part of the $z$-plane once, and each of the left and the right ones covers the lower part once. The point $H$ and $K$ are the ramification points.

As parameters vary and pass through the curve $\{D=0\}$, near the two confluenting ramification points, locally, the derived Schwarz map behaves similar to $\phi_{t}$.

2.5. Illustration of the image of the derived Schwarz map. We illustrate the behavior of the derived Schwarz map when the set of parameters traverses $D=0$. We study the (derived) Schwarz map with parameters $(a, b, c)=(1 / 2,1 / 2, c)$; we let $c$ move from 1 to 0 . In the st-plane in Figure 1, this move corresponds to the 

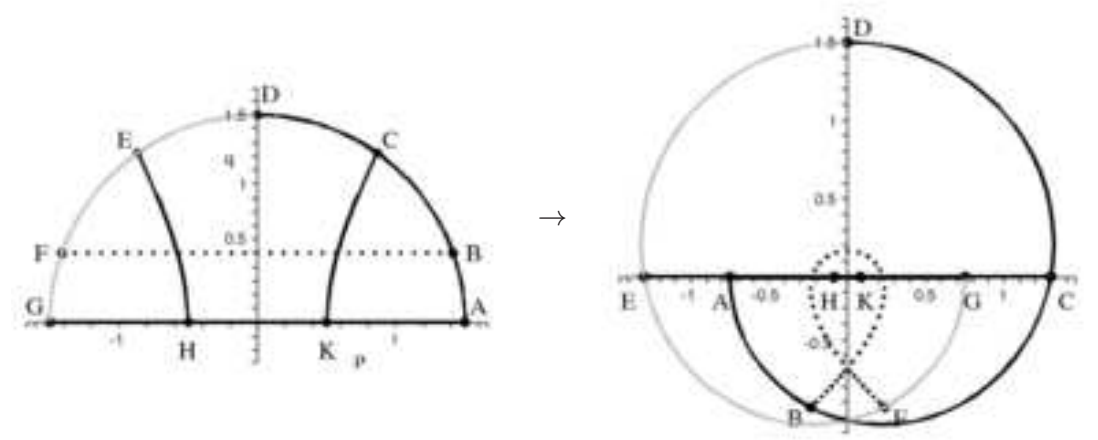

Figure 3 . The image of hemi-disc by $x \mapsto z=-x^{3} / 3+x / 4$

path along $t=s^{2} / 4$ starting at $(0,0)$; it crosses the curve $D=0$ at $(3 / 2,9 / 16)$ $(c=1-\sqrt{3} / 2)$, and goes out of the domain at $(2,1)$.

2.5.1. A normalization of the (derived) Schwarz map. In this subsection, for generic parameters $(a, b, c)$, we normalize the Schwarz map as

$$
S(0)=0, \quad S(1)=1, \quad \text { and } S(\infty)=\infty,
$$

The Schwarz map, in general, is multi-valued and determined by the equation $E(a, b, c)$ only up to conjugacy. Here we first choose two solutions

$$
u_{0}:=F(a, b, c, x) \quad \text { and } \quad u_{1}:=x^{1-c} F(a-c+1, b-c+1,2-c, x)
$$

in the upper half $x$-plane $X_{+}:=\{x \in C \mid \Im(x) \geq 0, x \neq 0,1\}$, where $F(a, b, c, x)$ denotes the hypergeometric function with parameters $(a, b, c)$. They are real valued on the interval $(0,1)$, since the parameters are real. We define temporarily a Schwarz map: $S_{T}(x):=u_{1} / u_{0}(x)$. The image of the interval $(0,1)$ is a real interval, $S_{T}(0)=$ 0 , and the image of the interval $(-\infty, 0)$ is a segment.

Lemma 2.6. If $a+b-c>0$, then

$$
S_{T}(1)=\frac{\Gamma(2-c) \Gamma(a) \Gamma(b)}{\Gamma(c) \Gamma(a-c+1) \Gamma(b-c+1)} .
$$

If $0<c<2$ and $a \leq b$, then

$$
S_{T}(\infty)=\frac{\Gamma(2-c) \Gamma(b) \Gamma(c-a)}{\Gamma(c) \Gamma(1-a) \Gamma(1-c+b)} e^{\pi i(1-c)} .
$$

Remark 2.7. Though the left hand-sides of the formulae are, by definition, symmetric in $a$ and $b$, the right hand-side of the second formula not.

Proof. (1) Consider solutions around $x=1$ :

$$
\begin{aligned}
& v_{0}:=F(a, b, a+b-c+1 ; 1-x) \text { and } \\
& v_{1}:=(1-x)^{c-a-b} F(c-a, c-b, c+1-a-b ; 1-x) ;
\end{aligned}
$$

set $s_{0}=u_{1} / u_{0}, s_{1}=v_{1} / v_{0}$. They are related as

$$
s_{0}=\frac{A s_{1}+C}{B s_{1}+D} \quad \operatorname{along}(0,1)
$$


where

$$
\begin{array}{rlrl}
D & :=\frac{\Gamma(c) \Gamma(c-a-b)}{\Gamma(c-a) \Gamma(c-b)}, & C:=\frac{\Gamma(2-c) \Gamma(c-a-b)}{\Gamma(1-a) \Gamma(1-b)} \\
B:=\frac{\Gamma(c) \Gamma(a+b-c)}{\Gamma(a) \Gamma(b)}, & A:=\frac{\Gamma(2-c) \Gamma(a+b-c)}{\Gamma(a-c+1) \Gamma(b-c+1)} .
\end{array}
$$

If $a+b-c>0$, then $s_{1}(1)=\infty$. Thus we have $s_{0}(1)=A / B$.

(2) We make use of Kummer's relations:

$$
\begin{aligned}
& u_{0}=(1-x)^{-a} F\left(c-b, a, c ; \frac{x}{x-1}\right), \\
& u_{1}=x^{1-c}(1-x)^{c-a-1} F\left(1-b, a+1-c, 2-c ; \frac{x}{x-1}\right),
\end{aligned}
$$

and let $x \rightarrow-\infty$. Then we have

$$
\frac{x^{1-c}(1-x)^{c-a-1}}{(1-x)^{-a}} \longrightarrow e^{\pi i(1-c)} \text { and } \frac{x}{x-1} \nearrow 1 .
$$

The Gauss formula

$$
F(a, b, c ; 1)=\frac{\Gamma(c) \Gamma(c-a-b)}{\Gamma(c-a) \Gamma(c-b)}, \quad \text { if } \quad c>0, c-a-b>0
$$

tells that, under the conditions $0<c<2$ and

$$
c-(c-b)-a=(2-c)-(1-b)-(a+1-c)=b-a>0,
$$

we have

$$
\begin{aligned}
\frac{F(1-b, a+1-c, 2-c ; 1)}{F(c-b, a, c ; 1)} & =\frac{\Gamma(2-c) \Gamma(b-a)}{\Gamma(b-c+1) \Gamma(1-a)} \cdot \frac{\Gamma(b) \Gamma(c-a)}{\Gamma(c) \Gamma(b-a)} \\
& =\frac{\Gamma(2-c) \Gamma(b) \Gamma(c-a)}{\Gamma(c) \Gamma(b-c+1) \Gamma(1-a)} \cdot
\end{aligned}
$$

We then define a new Schwarz map $S$ which sends 0,1 and $\infty$ to 0,1 and $\infty$, respectively, by

$$
S(x)=\frac{S_{T}(x)}{S_{T}(x)-S_{T}(\infty)} \frac{S_{T}(1)-S_{T}(\infty)}{S_{T}(1)} .
$$

Accordingly, the corresponding new derived Schwarz map $D S$ is defined: put

$$
U_{0}:=N \cdot u_{0}, \quad U_{1}:=N \cdot u_{1}, \quad D U_{0}:=\left(U_{0}\right)^{\prime}, \quad D U_{1}:=\left(U_{1}\right)^{\prime},
$$

and $D S_{T}:=D U_{1} / D U_{0}$, and we define

$$
D S(x)=\frac{D S_{T}(x)}{D S_{T}(x)-S_{T}(\infty)} \frac{S_{T}(1)-S_{T}(\infty)}{S_{T}(1)} .
$$

2.5.2. Illustration of confluence. We illustrate the images of the upper half plane under $S$ and $D S$ with parameters $(a, b, c)=(1 / 2,1 / 2, c), c=1, \ldots, 0.05$. Note that in this case,

$$
\mu_{0}^{2}=\mu_{1}^{2}=(1-c)^{2} \quad \text { and } \quad \mu_{\infty}^{2}=0 .
$$

Since $q=0$ is written as $x^{2}-x+\left(2 c-c^{2}\right)=0$, the discriminant is $D=4(1-c)^{2}-3$; we thus have

$$
\left.D\right|_{1>c \geq 1-\sqrt{3} / 2} \leq 0,\left.\quad D\right|_{1-\sqrt{3} / 2 \geq c>0} \geq 0 .
$$

Namely, when $c \geq 1-\sqrt{3} / 2$, the ramification point is located at

$$
\frac{1}{2}\left(1+i \sqrt{-4 c^{2}+8 c-1}\right)
$$

and when $c<1-\sqrt{3} / 2$, we have two ramification points

$$
\frac{1}{2}\left(1 \pm \sqrt{4 c^{2}-8 c+1}\right)
$$




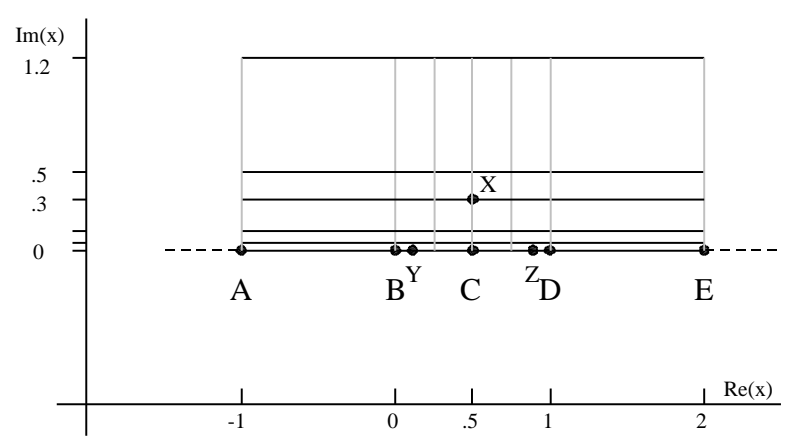

Figure 4.

on the real axis. Let us take a domain in the upper half plane as in Figure 4 , where $A=(-1,0), B=(0,0), C=(1 / 2,0), D=(1,0)$ and $E=(2,0)$, and the height of the quadrangle is $10 / 8$.

The point $X=(0.5, \sqrt{0.11}) \sim(0.5,0.3317)$ denotes the ramification point when $c=0.2$ and the points $Y=(0.5-\sqrt{0.1525}, 0) \sim(0.1095,0)$ and $Z=(0.5+$ $\sqrt{0.1525}, 0) \sim(0.8905,0)$ denote the ramification points when $c=0.05$. The images of these points under the derived Schwarz maps are bullets in Figures 5 and 6 with the same name. The bullets in Figure $5(c=0.90,0.50)$ without names are the (images of) ramification points.

Figures 5 and 6 show how the images of the domain under $S$ and $D S$ depend on the parameter $c$. In Figure 5 the image of the segments $\overline{A B}, \overline{B C}, \overline{C D}$ and $\overline{D E}$ under $D S$ is the segment $\overline{A B}$, the arc $B D C$, the arc $C B D$ and the segment $\overline{D E}$, respectively. However, in Figure 6 when $c=0.05$, the images of $\overline{B C}$ and $\overline{C D}$ are the curves

$$
B D Z C Y C \text { and } C Z C Y B D \text {. }
$$

In these figures, around $C$ (and $X, Y, Z$ ), we can observe the happenings described in Section 2.4 using the model map $\phi_{t}$.

In the case $c=1$, the inverse of the Schwarz map $S$ is the elliptic modular function $\lambda$, of which behavior is well-known. But the Schwarz map itself is a bit difficult to treat. The solutions $u_{0}$ and $u_{1}$ coincide, and the solutions $v_{0}$ and $v_{1}$ coincide. So the connection matrix relating these solutions loses its sense. Instead, we draw the associated surfaces in the 3 -ball as follows.

In order to get a total view of the images, we identify $\boldsymbol{P}^{1}$ with the boundary of 3-ball by the mapping

$$
\chi: \boldsymbol{P}^{1} \ni z \longmapsto\left(\frac{z+\bar{z}}{1+z \bar{z}}, \frac{-i(z-\bar{z})}{1+z \bar{z}}, \frac{z \bar{z}-1}{1+z \bar{z}}\right) \in \partial \boldsymbol{B}^{3},
$$

which is compatible with identification of $\boldsymbol{H}^{3}$ with the ball $\boldsymbol{B}^{3}$. In the case where $(a, b, c)=(1 / 2,1 / 2,1)$, the image of the upper half plane under $S$ is the fundamental domain relative to the triangular group usually denoted by $(\infty, \infty, \infty)$ that has the 


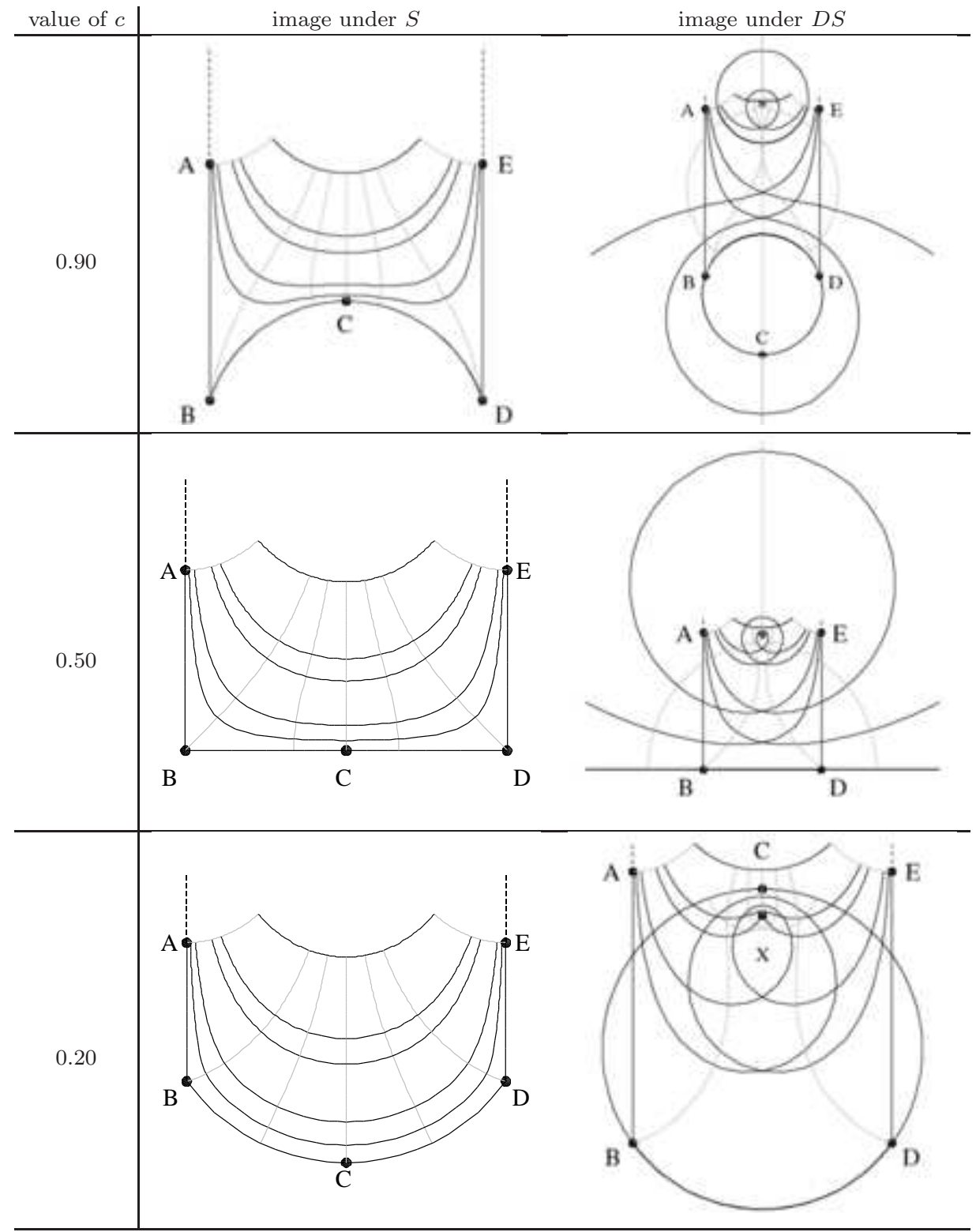

Figure 5. Images of the domain in Figure 4 under $S$ and $D S$ when $c>1-\sqrt{3} / 2$

picture in Figure 7. Its image in $\partial \mathbf{B}^{3}$ looks as in the left figure of Figure 8 . The image under $D S$ realized in $\partial \mathbf{B}^{3}$ is as in the right of Figure 8 . Here, the three gray-colored great circles on $\partial \mathbf{B}^{3}$ are added to evoke the reader a stereographic image.

\section{PARALlel FAMily OF FLAT FRONTS}

CONNECTING SCHWARZ AND DERIVED SCHWARZ MAPS

In this section, we study relations among the Schwarz map, the derived Schwarz map and the hyperbolic Schwarz map. 


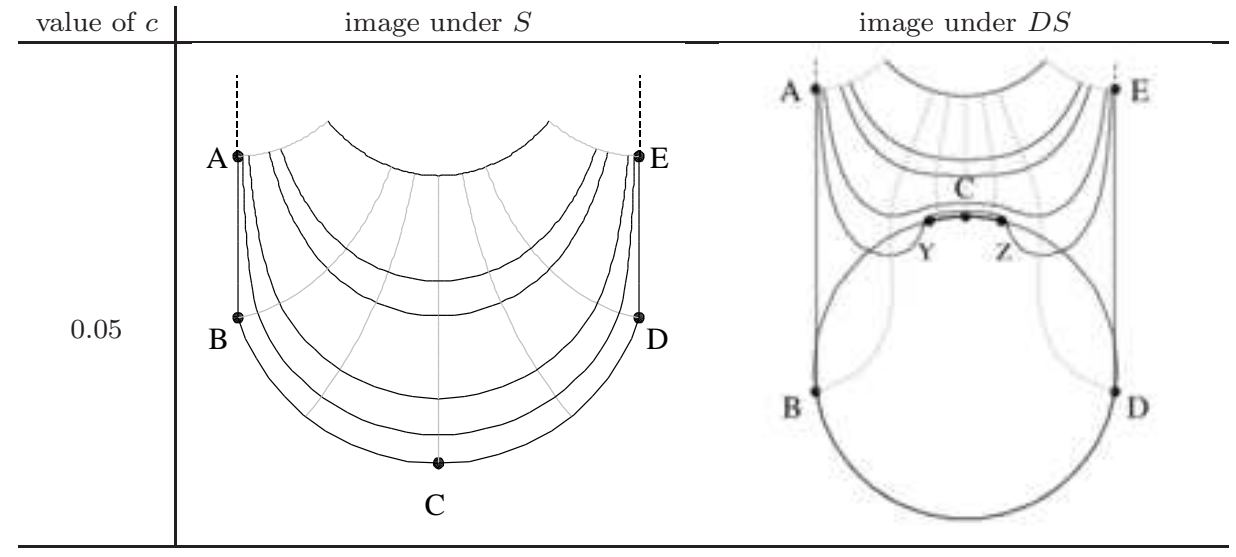

Figure 6. Images of the domain in Figure 4 under $S$ and $D S$ when $c=0.05(<1-\sqrt{3} / 2)$

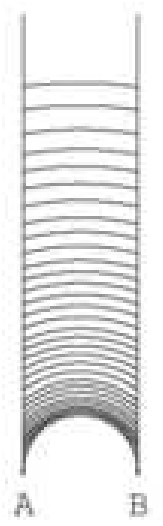

Figure 7 . The image $S\left(X_{+}\right)$
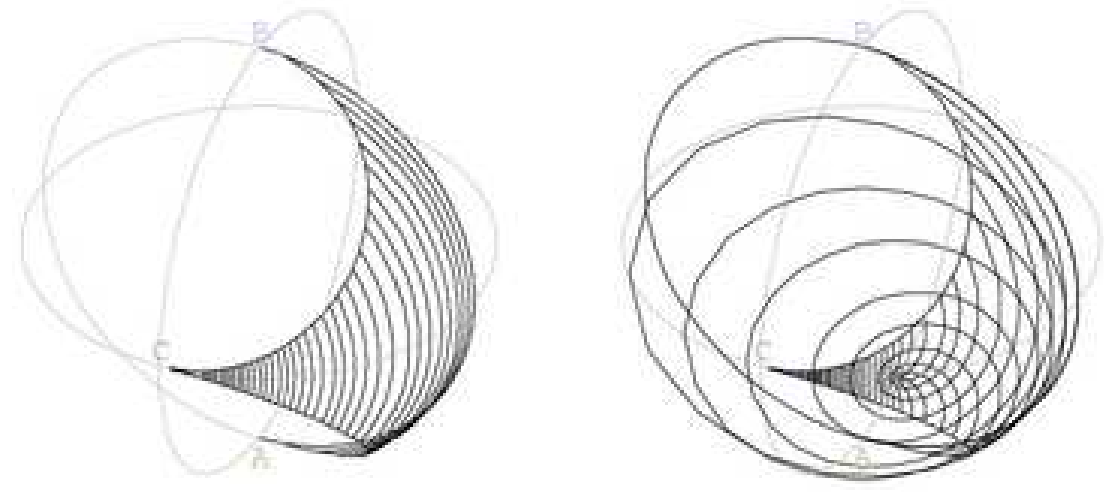

Figure 8 . The images $\chi\left(S\left(X_{+}\right)\right)$and $\chi\left(D S\left(X_{+}\right)\right)$ 
3.1. A relation between $S$ and $D S$. Since $S$ restricted to $X_{+}$is a biholomorphic isomorphism between $X_{+}$and a Schwarz triangle $T=S\left(X_{+}\right)$, we can consider the composite map $f:=\left.D S \circ S^{-1}\right|_{X_{+}}$. This map has a simple expression

$$
f: T \ni z \longmapsto z+2 \frac{\dot{x}}{\ddot{x}}
$$

where ${ }^{\cdot}=d / d z$. To show this expression, we let $u$ and $v$ be solutions of an $S L$ equation (SL) such that $u v^{\prime}-u v^{\prime}=1$. Since $z^{\prime}(:=d z / d x)=-1 / v^{2}$ and $\ddot{x}=$ $d^{2} x / d z^{2}$, we have $v=i / \sqrt{z}^{\prime}=i \sqrt{\dot{x}}, u=v z$, and

$$
u^{\prime}=i \frac{1}{\sqrt{\dot{x}}}+z \frac{i}{2}(\dot{x})^{-3 / 2} \ddot{x}, \quad v^{\prime}=\frac{d v}{d x}=\frac{d v}{d z} \frac{d z}{d x}=\frac{i}{2}(\dot{x})^{-3 / 2} \ddot{x} .
$$

Hence, we get (3.1).

Proposition 3.1. If $x(z)$ is invariant under $g \in P S L(2, \boldsymbol{C})$, that is, $x(g z)=x(z)$, then $f(z)$ is covariant under $g: f \circ g(z)=g \circ f(z)$.

Proof. Set

$$
g(z)=\frac{a z+b}{c z+d}, \quad a d-b c=1
$$

Then we have

$$
\dot{x}(g z)=(c z+d)^{2} \dot{x}(z), \quad \ddot{x}(g z)=(c z+d)^{4} \ddot{x}(z)+2 c(c z+d)^{3} \dot{x}(z) .
$$

Compute $f(g z)$ using these formula, we have the conclusion.

Remark 3.2. The covariance follows from the way of constructing the hyperbolic Gauss map, once we know that $S$ and $D S$ are the hyperbolic Gauss maps of the hyperbolic Schwarz map $H S$ (see Section 2.2).

3.2. Parallel family of flat fronts. As seen in Section 2.2, the hyperbolic Schwarz map is considered as a flat front

$$
\varphi:=H S=U^{t} \bar{U}: X \longrightarrow \boldsymbol{H}^{3}
$$

where $U: X \rightarrow S L(2, C)$ is the holomorphic lift as in (1.1).

The parallel front $\varphi_{t}$ of distance $t \in \boldsymbol{R}$ of the flat front $\varphi$ is defined as

$$
\varphi_{t}(x)=\exp _{\varphi(x)} t \nu(x)=(\cosh t) \varphi(x)+(\sinh t) \nu(x),
$$

where $\nu$ is the unit normal vector field as in (2.1) and exp denotes the exponential map of $\boldsymbol{H}^{3}$. In the second expression of (3.2), we identify $\boldsymbol{H}^{3}$ with the upper half component of the two-sheet hyperboloid in the Minkowski 4 -space. Then the parallel front is expressed as

$$
\varphi_{t}=U_{t}{ }^{t} \overline{U_{t}}, \quad U_{t}=U\left(\begin{array}{cc}
e^{t / 2} & 0 \\
0 & e^{-t / 2}
\end{array}\right),
$$

see [5], in which the holomorphic lift is denoted by " $E$ ". The hyperbolic Gauss maps of $\varphi_{t}$ coincide with those of $\varphi$ (which are $G=S$ and $G_{*}=D S$ in our case), and the normal geodesic starting at $\varphi(x)$ intersect with the ideal boundary at $G(x)=S(x)$ and $G_{*}(x)=D S(x)$.

The induced metric (the first fundamental form) of $\varphi_{t}$ is expressed as

$$
d s_{t}^{2}=q_{t} d x^{2}+\bar{q}_{t} d \bar{x}^{2}+\left(1+q_{t} \bar{q}_{t}\right) d x d \bar{x}, \quad \text { where } \quad q_{t}=e^{t} q .
$$

Set $r(x):=-\log |q|$. Then $\varphi_{r(x)}$ has a singularity at $x$. Hence the locus of the singular points of $\varphi_{t}$ is expressed as

$$
\psi(x)=\varphi_{r(x)}(x),
$$



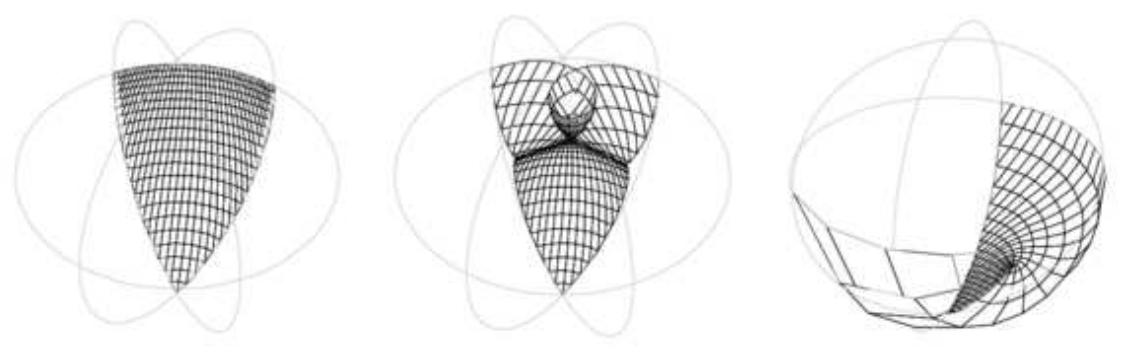

Figure 9. The images $\chi\left(S\left(X_{+}\right)\right), H S\left(X_{+}\right)$and $\chi\left(D S\left(X_{+}\right)\right)$
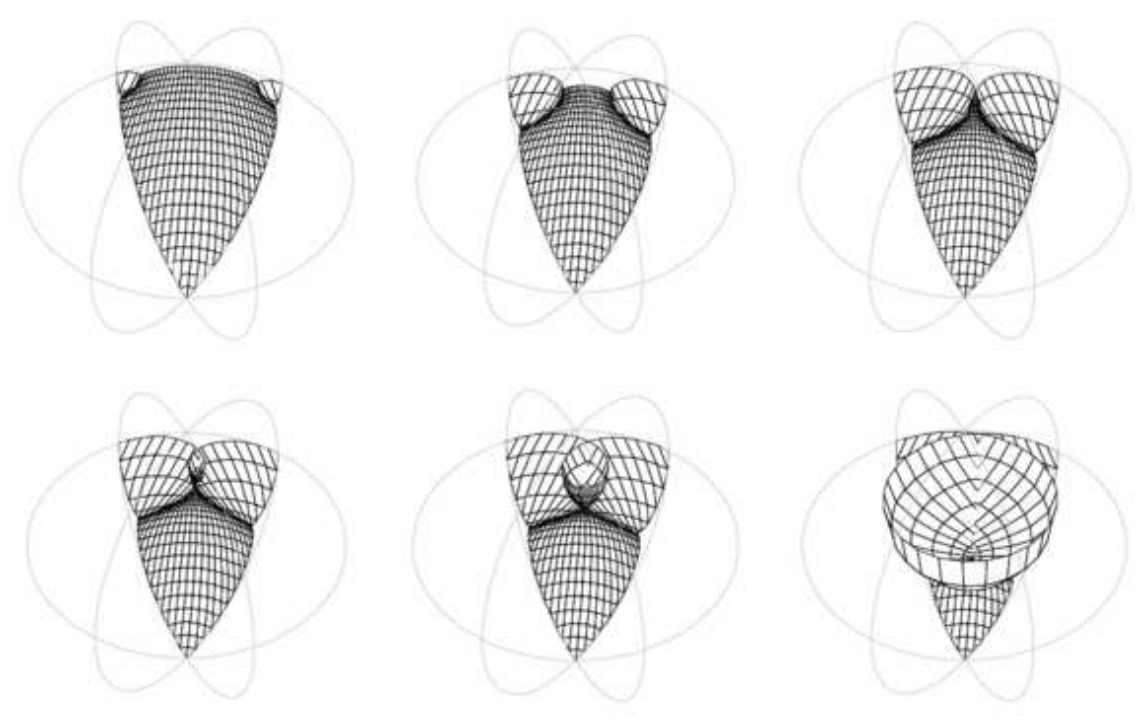

Figure 10. Parallel family of the images when $(a, b, c)=(1 / 6,-1 / 6,1 / 2)$

which is the caustic of the front $\varphi$. It is known that, locally, the caustic of the flat front is a flat front, see [6]. More detailed discussions are found in [4]; refer also to [1, 5, 3] for the materials above.

3.3. View of the parallel family. Relying on the previous discussion, we draw pictures in the case where $(a, b, c)=(1 / 6,-1 / 6,1 / 2)$, i.e. the case where the monodromy group is the dihedral group of order 12 ; refer to 7 .

First, the domain $S\left(X_{+}\right)$is a fan with center at $(0,0)$ of radius 1 with angle from 0 to $\pi / 3$. Its image in the sphere, $\partial \boldsymbol{B}^{3}$, is drawn in the left of Figure 9 The right two figures are the image $H S\left(X_{+}\right)$and $\chi\left(D S\left(X_{+}\right)\right)$; here, one half of $\chi\left(D S\left(X_{+}\right)\right.$ is drawn for the sake of a better view.

Second, the parallel family to $H S\left(X_{+}\right)$is drawn in Figure 10 from left to right and from top to down, the figures gradually change their shape from $\chi\left(S\left(X_{+}\right)\right)$to $\chi\left(D S\left(X_{+}\right)\right)$.

Third, Figure 11 (left) draws the section by the equatorial plane of the parallel flat fronts. The gray curves are geodesics joining $\chi\left(S\left(X_{+}\right)\right)$and $\chi\left(D S\left(X_{+}\right)\right)$. Here, $\chi\left(S\left(X_{+}\right)\right)$is the arc $\widehat{X Y}$, and $\chi\left(D S\left(X_{+}\right)\right)$is the arc starting at $X$, going around the circle once and then terminating at $Y$; thus the arc $\widehat{X Y}$ is covered twice. Figure 11(Right) is an enlargement of the left. The enveloping curve of geodesics is the 

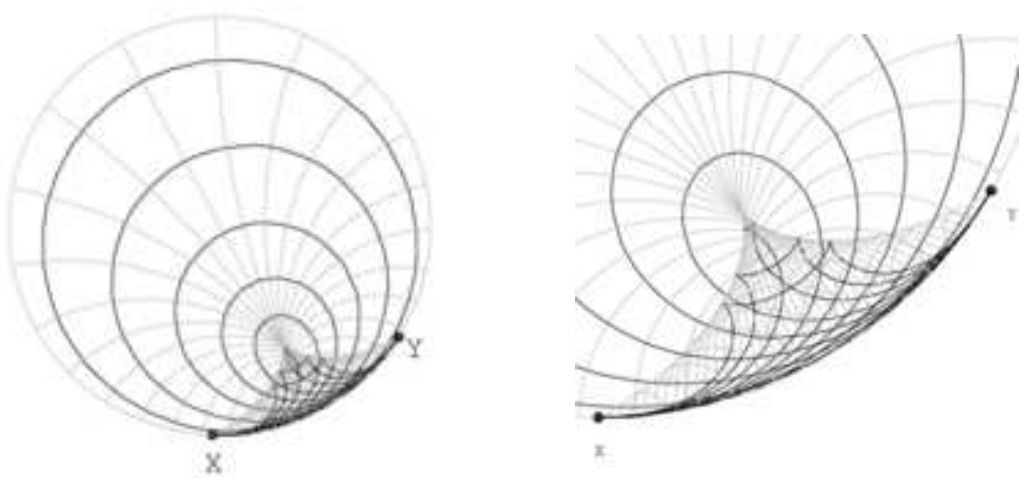

FiguRE 11. Left:The section by the equatorial plane of the parallel family, Right:Caustic locus

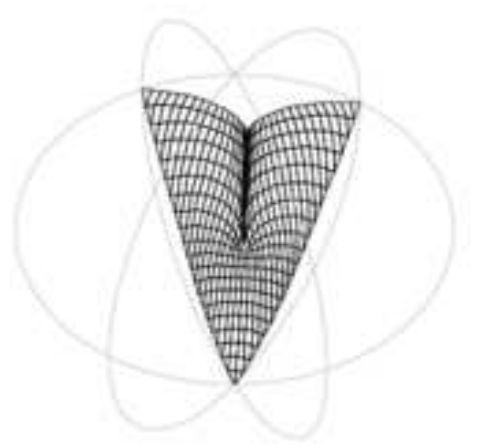

Figure 12. View of the caustic locus

section of the caustic locus, where some of sections of the flat fronts have cuspidal edges. Figure 12 gives a total view of the caustic locus.

\section{REFERENCES}

[1] J. A. Gálvez, A. Martínez and F. Milán, Flat surfaces in hyperbolic 3-space, Math. Ann. 316 (2000), 419-435.

[2] K. Iwasaki, H. Kimura, S. Shimomura and M. Yoshida, From Gauss to Painlevé - A MODERN THEORY OF SPECIAL FUnCTIONS, Vieweg Verlag, Wiesbaden, 1991.

[3] M. Kokubu, W. Rossman, K. Saji, M. Umehara and K. Yamada, Singularities of flat fronts in hyperbolic 3-space, Pacific J. Math. 221 (2005) 303-351.

[4] M. Kokubu, W. Rossman, M. Umehara and K. Yamada, Flat fronts in hyperbolic 3-space and their caustics, J. Math. Soc. Japan 59 (2007), 265-299.

[5] M. Kokubu, M. Umehara and K. Yamada, Flat fronts in hyperbolic 3-space, Pacific J. Math. 216 (2004) 149-175.

[6] P. Roitman, Flat surfaces in hyperbolic 3-space as normal surfaces to a congruence of geodesics, preprint.

[7] T. Sasaki, K. Yamada and M. Yoshida, Hyperbolic Schwarz map for the hypergeometric equation, preprint, math.CA/0609196

[8] H. Shiga, T. Tsutsui and J. Wolfart, Triangle Fuchsian differential equations with apparent singularities; with an appendix by P. B. Cohen, Osaka J. Math. 41(2004), 625-658.

[9] M. Yoshida, Hypergeometric Functions, My Love, Vieweg Verlag, Wiesbaden, 1997. 
(Sasaki) Department of Mathematics, Kobe University, Kobe 657-8501, Japan

E-mail address: sasaki@math.kobe-u.ac.jp fax number:+81-78-803-5610

(Yamada) Faculty of Mathematics, Kyushu University, Fukuoka 812-8581, JAPAN

E-mail address: kotaro@math.kyushu-u.ac.jp

(Yoshida) Faculty of Mathematics, Kyushu University, Fukuoka 810-8560, Japan

E-mail address: myoshida@math.kyushu-u.ac.jp 